\title{
The impact of space and time on interprofessional teamwork in Canadian primary health care settings: implications for health care reform
}

\author{
Ivy F. Oandasan ${ }^{1}$, Lesley Gotlib Conn ${ }^{2}$, Lorelei Lingard ${ }^{3}$, Allia Karim², Difat Jakubovicz ${ }^{4}$, \\ Cynthia Whitehead ${ }^{5}$, Karen-Lee Miller ${ }^{6}$, Natalie Kennie ${ }^{7}$ and Scott Reeves ${ }^{8}$ \\ ${ }^{1}$ Director, Office of Interprofessional Education; Associate Professor and Research Scholar, Department of Family \\ and Community Medicine; Faculty of Medicine, University of Toronto; Academic Family Physician, Family Health \\ Centre (FHC), Toronto Western Hospital, Toronto, Ontario, Canada \\ ${ }^{2}$ Research Associate, Office of Interprofessional Education, at the University Health Network, Toronto Western \\ Hospital, Toronto, Ontario, Canada \\ ${ }^{3}$ Associate Professor, Department of Pediatrics, University of Toronto; Scientist, Wilson Centre for Research in \\ Education at the University of Toronto, Toronto, Ontario, Canada \\ ${ }^{4}$ Assistant Professor, Department of Family and Community Medicine, University of Toronto; Staff Physician, \\ St Joseph's Health Centre, Toronto, Ontario, Canada \\ ${ }^{5}$ Assistant Professor, Department of Family and Community Medicine, University of Toronto; Staff Physician, \\ Women's College Hospital, Toronto, Ontario, Canada \\ ${ }^{6}$ Research Associate, Li Ka Shing Knowledge Institute (at time of research); Research Associate, Public Health \\ Agency of Canada; PhD candidate in the Dalla Lana School of Public Health, University of Toronto, Toronto, \\ Ontario, Canada (currently) \\ ${ }^{7}$ Primary Care Pharmacist, St Michael's Hospital, Toronto, Ontario, Canada \\ ${ }^{8}$ Scientist, Li Ka Shing Knowledge Institute of St Michael's Hospital; Director of Research, Centre for Faculty \\ Development, St Michael's Hospital; Scientist, Wilson Centre for Research in Education; Associate Professor, \\ Department of Psychiatry, University of Toronto, Toronto, Ontario, Canada
}

\begin{abstract}
Aim: This paper explores the impact of space and time on interprofessional teamwork in three primary health care centres and the implications for Canadian and other primary health care reform. Background: Primary health care reform in Canada has emphasized the creation of interprofessional teams for the delivery of collaborative patient-centred care. This involves the expansion and transformation of existing primary health care centres into interprofessional family health teams (FHT) promising to provide patients better access, more comprehensive care, and improved utilization of individual health professionals. Benefits for providers include improved workplace satisfaction and organizational efficiencies. Currently, there is little evidence for how effective interprofessional teamwork happens and little is known about how to create high-functioning teams in the primary health care setting. Methods: We used ethnographic observations and interviews to gain a deep understanding of the nature of interprofessional teamwork. Three academic family health centres participated in a total of $139 \mathrm{~h}$ of observation and 37 interviews. Team members in all three centres from the disciplines of medicine, nursing, physiotherapy, occupational therapy, social work, dietetics, pharmacy, and office administration participated in this study. Findings: We found that both the quantity and quality of interprofessional communication and collaboration in primary health care is significantly impacted by space and time.
\end{abstract}

Correspondence to: Ivy F. Oandasan (MD CCFP MHSc FCFP), Office of Interprofessional Education at the University Health Network, Toronto Western Hospital, 750 Dundas Street West, Suite. 302 Toronto, Ontario M6J 3S3. Email: ivy.oandasan@uhn.on.ca

(C) 2009 Cambridge University Press 
Across our research sites, the physical layout of clinical space and the temporal organization of clinical practice led to different approaches to, and degrees of success with, interprofessional teamwork. Varied models of interprofessional collaboration resulted when these factors came together in different ways. These findings have important implications for the transition to interprofessional family health teams in Canada and beyond.

Key words: communication; ethnography; interprofessional teamwork; primary health care; space; time

Received: 18 June 2008; accepted: 10 March 2009

\section{Background}

Building on international advances, specifically the United Kingdom's National Health Service (Department of Health, 2000; Department of Health, 2004), recent Canadian primary health care reform has centred on the development of interprofessional teams for a more effective delivery of care (Health Canada, 2004). This effort targets processes of health professions' collaboration to improve patient care, provider satisfaction, and organizational efficiencies by creating new Family Health Teams (FHT). The FHT model marks a transition from the traditional model of uniprofessional physician-based care to a team-based approach in which family physicians, nurses, pharmacists, social workers, and other health care providers will work collaboratively to ensure that care can be coordinated and delivered in a seamless manner (Meuser et al., 2006). This interprofessional approach that underpins the new emerging FHT model is promised to result in 'more comprehensive care being provided to patients' and the opportunity to use health care professionals 'to the maximum extent of their capabilities' (Ontario Ministry of Health and Long Term Care, 2002).

To date, we know little about how effective interprofessional teamwork is enacted in hospital settings generally (Reeves and Lewin, 2004), and in family practice settings in particular. Recent literature on the creation of interprofessional teams in primary care in the UK calls attention to the fact that team formation is developmental (Shaw et al., 2005), and team members require continuing education and contextually relevant training to successfully collaborate (Boudioni et al., 2007). A study by Soklaridis et al. (2007) in a Canadian academic family health centre reveals that significant barriers to interprofessional team building and cohesion occur because of a lack of role understanding amongst health professionals and a paucity of existing processes to teach collaboration. This is consistent with findings by Wilson et al. (2005) that Canadian family physicians hold a positive view of interprofessional collaboration but believe several barriers, including education around interprofessional team building, must be overcome for such work to be actualized. Furthermore, a recent synthesis report on interprofessional collaboration and quality primary health care in Canada reveals that, despite reported positive outcomes of initiatives promoting interprofessional collaboration, there is no literature to date comparing how different models of interprofessional collaboration affect client, provider, and system outcomes (Barrett et al., 2007). More research is therefore needed to better understand how primary care providers can effectively prepare and organize to provide seamless interprofessional care.

\section{Research on space and time in health care}

Researchers in health geography are engaged in debate about approaches to understanding the relationship between place, space, and the health care system. Advancing a framework for geographies in health care, Andrews and Evans (2008) argue that research on the spatial dimensions of health care to date has focused very much on the consumption of the health care system and very little on its production. With the exception of work from within the field of nursing (Liaschenko, 1994; Halford and Leonard, 2003; Andrews, 2006) 
there is a relatively small amount of research examining how health care workers produce the health care system via the organization of their day-to-day practices. Little is known about how workers' spatial organization impacts health care team composition and dynamic, the construction of professional identities, and the establishment of workers' professional boundaries, roles, and responsibilities (Andrews and Evans, 2008). What little recent research in the primary health care setting we have is from Rapport et al. (2006; 2007) who focus on general practitioners' (GPs) relationships between their workspaces and professional practices in the UK. Rapport et al. (2006) report GPs' negative reflections on such aspects as the unsuitability and poor organization of their clinics for examining patients, as well as positive reflections on the personalization of clinical space and how it can be strategically used to assess patients. While insightful for understanding how space influences physicians' morale and physician-patient interactions, additional research is needed which could explore space in an interprofessional setting and its implications for the construction of relationships between physicians and other health care providers, and their collaborative work.

Some recent health services research explores various dimensions of time as a factor impacting health service delivery. For example, how primary health care physicians' allocate time spent with patients (Tai-Seale et al., 2007), physicians' time constraints for the management of chronic illness and prevention (Yarnall et al., 2003; Ostbye et al., 2005), and time as a barrier to physician engagement in primary health care research (HummersPradier et al., 2008). Hansson et al.'s (2008: 9) case study of four interprofessional teams indicates GPs experience teamwork as both 'time-consuming' and 'time-saving,' which lends to their feelings of ambivalence about collaborating with other health care providers. According to DS Thompson et al. (2008: 540), nursing researchers routinely report on the perceptions and impact of nurses' lack of time for effective clinical practice. Recent Canadian nursing research examines the construction of a 'culture of busyness' for nurses and its implications for their use of research (DS Thompson et al., 2008: 545), as well as the negative effects of time pressure on nurses' decision-making in acute care (C Thompson et al., 2008). Still, research is limited on the real or perceived influence of time on the production of other primary health care providers' work specifically on interprofessional teams.

In this paper, we present findings from a qualitative study of interprofessional team collaboration in three academic primary health care centres. This research was carried out as part of the Structuring Communication Relationships for Interprofessional Teamwork (SCRIPT) Programme, a multi-site, multi-method project dedicated to transforming the culture of interprofessional practice within academic clinical teaching units in general internal medicine, primary care, and rehabilitative care (Reeves et al., 2007). Here, we argue that both the quantity and quality of interprofessional communication and collaboration in primary health care is significantly impacted by space and time. Across our research sites, the physical layout of clinical space and the temporal organization of clinical practice led to different approaches to, and degrees of success with, interprofessional collaboration and teamwork. We find varied models of interprofessional collaboration result when these factors come together in different ways. These findings have important implications for the design and success of future FHTs in Canada and beyond.

\section{Methods}

\section{Setting and participants}

Staff in three academic family health centres participated in our research. We selected these sites because they were primary health care units involved in interprofessional collaboration and would be transitioning to become FHTs under the new health system reform initiatives. In addition, various staff members in each of these sites were well known to members of the SCRIPT research team and expressed interest in participating in the research. Their a priori willingness to endorse and participate in the study was a necessary first step to gaining access to the family health centres and approval from the research ethics boards of each site.

\section{Site 1}

Site 1 was a medium-sized practice located in a community teaching hospital. It served a culturally 
and linguistically diverse patient population, offering specialized services in obstetrics, palliative care, addiction, and mental health. In addition to management personnel (department head, patient care coordinator, and team leader) it comprised 14 staff physicians, 22 medical residents, 18 medical students, five nurses (two full-time, one part-time, two casual), a dietitian, a behavioural science educator, and nine clerical staff. An administrative staff of three was also responsible for clinic scheduling, the administration of the medical training programmes, as well as the palliative and addiction medicine programmes.

\section{Site 2}

Site 2 was a large urban practice located in an ambulatory care hospital. Its patient population included young professionals, families, and seniors. It also had a particular focus on low-risk obstetrical care. The clinic was divided into seven core teams, each comprised of part-time staff physicians, residents, a nurse, and a secretary. The number of physicians on each team ranged from two to eight for a total of 27 , as did the number of residents and medical students for a total of about 24 for each group, each year. Other health care providers included a nurse practitioner, dietitian, social worker, occupational therapist, and physiotherapist. These individuals were not affiliated with any one team but serviced the entire clinic. There was also a clinical manager, department head, and one unit-wide administrative assistant responsible for general clinic inquiries and booking new patients.

\section{Site 3}

Site 3 was a relatively small practice affiliated with a large inner-city hospital. It served a generally poor and disadvantaged population. The clinic saw patients with a range of issues from annual checkups to HIV/AIDS treatment. There were two teams in the clinic; one was staffed by four physicians and one nurse, and the other by three physicians and one nurse. Three family medicine residents were also associated with the two teams. Other health care providers who serviced the entire unit, as well as other primary health care centres affiliated with the same hospital included a social worker, dietitian, pharmacist, occupational therapist, addictions counsellors, and two lab technicians. There were nine clerical assistants who worked with both physician-nurse teams. Other on-site personnel included a clinical leader manager and administrative manager.

\section{Data collection}

Data were collected using ethnographic observations and interviews in the clinical settings. Ethnographic methods render rich understandings of people and social processes by considering how behaviours, beliefs, and actions are made meaningful in local settings (Hammersley and Atkinson, 1995). Firstly, we observed staff as they participated in their daily activities focusing on the organization, purpose, location, and type of interactions that promoted or limited interprofessional collaboration. Data was gathered by four social science researchers who collectively completed approximately $50 \mathrm{~h}$ of observation in each of the three sites over the course of five months. Positioned in communal areas on the unit, such as the reception desk, the observers recorded fieldnotes by hand. Observational data was also recorded at meetings. The researchers did not record interactions between staff and patients. They did not have direct access to patient charts but observed the use of charts and other patient records by health care staff. Reflective fieldnotes were also written following observations. A coding system was employed to number participants by profession (e.g., MD1) for anonymity.

Secondly, we undertook 37 individual interviews with a range of team members. Participants were purposively selected to reflect the perspectives of different professions and their varying responsibilities. Participants were asked to describe their roles in their workplace, their work environment, and any barriers and facilitators to collaboration. Researchers took notes during the interviews and later transcribed them into more descriptive fieldnotes which included their reflections (Sanjek, 1990).

Institutional research ethics approval was obtained from all relevant institutions. Individual staff and trainees in each of the sites were asked for their oral consent for participation prior to, and throughout, the observations. In the case of interviews, we obtained written consent from individuals. No staff declined participation in the research. Patients were notified about the presence 
of researchers in the clinics by way of information notices posted in high-traffic areas. These notices included photos of the researchers and invited patients to ask questions about the study.

\section{Data analysis}

Inductive data analysis was conducted through an iterative approach, in which analysis occurred alongside data collection and informed it. The research team, made up of clinicians, qualitative researchers, and educators (all authors on this article), met regularly to discuss emerging trends in the data, collection strategy, and analytical issues. Researchers also met with appointed on-site investigators from each clinic to explore the resonance of emerging findings and seek direction regarding exploration of outstanding issues. Data collection ended when the research team and co-investigators agreed that saturation had been reached, that is, that no new themes were emerging from the data and that we had captured a representative view of interprofessional collaboration in each site. Using a constant comparative approach, salient instances in the fieldnotes were examined to construct categories representative of recurring themes. One feedback session, that included staff members, was held at each of the sites to return findings for elaboration and refinement.

\section{Results}

Our findings are organized in three sections. First, we provide a general description of team structure and clinical configuration as observed in each site. Second, we present data on the impact of space. We highlight how the co-location of team members and availability of space for interpersonal interactions were important factors for relationship-building and establishing team cohesion. Third, we present our data on the impact of time on interprofessional collaboration across the three sites. We draw attention to the effects of busy clinical schedules and length of time worked in the clinic on participants' experiences of time as a barrier to communication and teamwork. In the discussion, we compare and describe how time and space together led to different approaches to teamwork in each of our sites, illustrating their interdependent and differ- ential effects on the organization and experience of interprofessional work.

The terminology we use to describe the participants of the study is chosen to provide consistency and clarity. We recognize that other terms have been used in the literature. For the purposes of this paper, we will distinguish physicians from other health care professionals (which will include nurses, social workers, pharmacists, etc.) and administrative staff (which will include receptionists and secretaries).

\section{Structural layout}

Site 1 operated as one multi-professional unit. Clerical staff worked with all physicians and nurses to book patients, complete consultation requests, file charts, etc. The physical layout of the clinic facilitated this practice design: clerical staff shared a small and often over-crowded reception and filing area close to the centre of a hallway. Down each side of the hall, physicians had personal offices that doubled as examination rooms. Next to the reception area was a semi-enclosed nursing station and further down the hall were trainee supervision rooms. Both the reception and nursing areas were key communication sites where quick, unstructured interprofessional work occurred. There were two patient waiting areas; a larger one for the majority of patients was located outside the actual clinic doors across a short hallway. A small inner clinic waiting room sat approximately seven patients who were typically elderly. The dietitian and behavioural science educator did not have offices within the clinic itself. At the time of our research, offices of administrative staff and the department head had been relocated to an area on the other side of the hospital. This was due to the renovations that had begun expanding the clinic for its transition to a FHT.

In site 2, seven core teams were each assigned their own space within the larger unit; six of these were within close proximity on the same floor of the building, while one was on another floor in an adjacent building. Within each of these microclinics the secretary occupied the key communication area. Her personal open workspace faced a row of chairs for waiting patients. There were two or three examination rooms for each team, as well as a private office for the nurse and a shared 
office space for physicians. Patient charts were held in the basement of the building and retrieved and distributed throughout the day by health records staff. Other health care professionals, such as the social worker, were located on a different floor than the majority of the clinical teams. During our research, renovations had begun to expand the one existing team and relocate non-medical health professionals to a more populated floor, again for the transition to a FHT.

Site 3 occupied one half of the ground floor of a medical office building. Clerical staff shared a comfortable-sized reception area at the clinic's entryway where patient charts were also stored. Doctors' and nurses' personal offices, exam rooms, a patient waiting room, and the laboratory were closed-off spaces along the four short hallways of the square-shaped unit. The offices of non-medical health professionals were on a different floor of the building as was a shared meeting room. Site 3 had recently moved from an entirely different clinical space where some members of the non-medical allied health staff were co-located among physicians, nurses, and administrative clerks. The former clinic, though somewhat rundown and isolated, had housed the group for over a decade.

\section{The impact of space}

In conversation with researchers, participants referred, often and unprovoked, to the undesirable physical layout of their clinics, commenting on the negative impact of 'poor design' (site 2, FN 01) and 'cramped office[s]' (site 3, FN 19) on patient privacy and administrative productivity. As observers, we experienced the constraints of space on communication first hand as we attempted to carve out unobtrusive but central space of our own to accurately capture interprofessional communication. This proved to be challenging in all sites, given the small reception areas that were already crowded. Yet, we found that space was used creatively to overcome the limits faced. In site 2 , a nurse rarely did her administrative work in her own private office, preferring the space next to the secretary in the reception area, which she felt was less confining. When asked by an observer about her choice she replied, 'Have you seen my office? It's an old utility closet. It has no windows or air circulation' (FN 16). Her makeshift workspace gave her easier access to the computer and incoming phone calls from patients, as well as enabled her to have 'an ear to the secretary's needs' (FN 16). With clear benefits for both the nurse and secretary, spatial barriers in this instance were overcome.

Physicians, nurses, and clerical/secretarial staff worked physically close to one another in each of the three sites. Yet, distance of some interprofessional staff from one another was a noted spatial impediment to communication and collaboration. Where nursing and clerical staff were not assigned or did not create shared workspace, collaboration was not easily achieved. In site 1, the physically separated workspaces of nurses and clerks meant that nurses experienced influxes of phone calls that were at times inappropriately triaged and unmanageable. Frustrations with this were sometimes directed at the clerical group. As one nurse frankly explained, 'The problem is that nurses and clerks are not working directly with each other. [I] would like to have some of the clerks [working] in the nursing station' (FN 22).

For other health care professionals in each of the three sites, co-location was a foremost barrier to interprofessional collaboration and feeling like part of a larger team. In site 2, for example, offices of the social worker, physiotherapist, and dietitian were separate from the main clinical area, though in the same building. According to a dietitian, despite working in the clinic for 14 years, it was 'interesting being a single entity' (FN 14). Feeling as though the clinic operated in professional silos, she hoped the transition to a FHT and its accompanying renovations would change this. A physiotherapist from site 2 confirmed that, 'If she were in the same space as the [core] teams, she would [have] contact with them more often. Because of her geographical location her communication with [the teams] was limited and infrequent' (FN 03).

When other health care professionals were entirely off-site some experienced an even greater sense of exclusion from the main clinical team. This was partly the result of having to travel, under time constraints, to participate in team activities. An occupational therapist in site 3 explained that she had had a hard time making it to the team meetings when they were (in the former location) because it was an effort to get there and the building was locked if she did not make it right on time. She did not feel 'part of the 
original team' and attributed this to distance and the minimal contact she had had historically with the interprofessional group (FN 19).

At the time of our research, the staff in site 3 were in a unique position to reflect on the changes to interprofessional collaboration that were influenced by the layout of their new clinic. Previously, most staff worked and ate lunch in one shared space, and felt the constant visibility and access to one another had contributed to the development of an inclusive collaborative team environment. Upon moving to their new clinic six months before our research began, this changed for some whose offices were no longer within the core clinical area. Other health care professionals who were previously on-site were now located on the third and fourth floors of the new building, while the physicians, nurses, and administrative staff worked on the first floor. A social worker who had worked in this group for 14 years remarked,

I think the docs that I was physically close to I had more contact with. I was embedded in the team, present, and it shifted the culture. I was around more and could have more impact, even in informal conversation, like the lunch room... The elevator here, which divides [me] from the rest of the clinic, has changed [my] interactions. At the previous site...[I] was very close to other people.

(FN 01)

The development and maintenance of interprofessional relationships was partly contingent upon the availability of space for informal opportunistic communication. Some of the easily observable barriers to opportunistic communication, in fact, were attributable to the way clinical space was amenable or not toward this end. For instance, in site 2 , each of the seven core teams occupied a semi-enclosed physical area, which was well designed for the functioning and cohesion of the small autonomous physician-nursesecretary teams. Yet, regular impromptu intersection across the core teams was not the norm; staff members were observed making few visits to one another in their respective sub-clinics, and in most cases would not spontaneously see one another during clinic hours. This meant some staff in the larger unit of the same and different professions did not really know each other and lacked some knowledge of the other professionals' roles. One secretary explained that generally 'There's not a lot of communication that happens with other teams' (FN 05). And as far as informal conversation between the secretaries and nurses was concerned, the same secretary stated,

We have a lunchroom downstairs where the secretaries will all go and chit chat with one another...the nurses go over to the government building. They circle among themselves. I'll say hi to them when I see them but not much interaction happens otherwise.

(FN 05)

Reflecting nostalgically on the spatial advantages of their former clinic, staff in site 3 described having had a meaningful connection to the space itself, which, in turn, connected them to one another regardless of their professions. The informal design of the space led to a sense of solidarity among those who were originally co-located in the clinic. A physician described the informality of the group as being 'defined' by the clinic, which was 'not very clinical' in its appearance (FN 15). According to a clerical worker, staff in site 3 'worked better as a team at the old location' which 'was not as professional' (FN 16). One health care professional reported that the change in their relationships had even been noted by 'the cleaning person' who told him 'it's not the same here...there was a family feeling over there' that came from use of that space (FN 01).

\section{The impact of time}

The organization of clinical time played a meaningful role in shaping interprofessional communication and collaboration. We discovered a common pattern of communication largely influenced by patient care schedules and accompanying time pressures and constraints. Daily communication between the professions was mostly unstructured and unplanned. Staff did not hold daily meetings where they discussed patients or administrative issues. Doctors, nurses, and clerical staff interacted on an ad hoc basis during the one- to five-minute transitions from one scheduled patient appointment to the next. Exchanges were consequently brief and focused, mainly face-to-face or by telephone. Since the 
reception area was the hub of activity in each of the units, the majority of interprofessional communication happened there. It sometimes took place spontaneously in open areas, such as utility or supply rooms, as well as in hallways.

Participants from within all professions recognized that time, or lack thereof, posed a challenge to having substantial daily interprofessional interactions from which they believed they would benefit. In site 1 , the team leader stated that they 'need to have a group interaction where they can say what's going on today, just for ten minutes or so' (FN 06). According to a physician, this 10-minute daily session had potential for mending the 'communication breakdown between clerks and medical staff' that has persisted for 'a long time' (FN 06). In sites 1 and 2, such scheduled or formalized interprofessional discussions happened only on a monthly basis which many participants believed was not frequent enough. For example, when we asked a nurse in site 2 if she would like to see any different opportunities in place to improve communication on her team, she replied that 'meetings would be great to discuss issues... but the timing is hard, especially because two of the doctors on this team are part time' (FN 16). A physiotherapist in site 2 agreed that 'formal communication' was a great idea 'theoretically, however, 'there is not enough time' the way the clinic is currently organized (FN 03).

By comparison, site 3 had a weekly interprofessional patient care meeting that had been ongoing for at least a decade. This gathering was highly valued by staff for providing a lengthy opportunity for communication about patients and problem-solving. Physicians, nurses, nonmedical allied health staff, and representatives from the clerical staff gathered over lunch in a common meeting room; sessions were chaired on a rotating basis by the nurses. These meetings were a successful form of communication because, as one nurse explained,

In addition to discussing [patient] cases, other issues can be brought up, for example, logistical issues, like if [doctors] had patient flow problems.

(FN 13)

Though some allied health staff felt as though these meetings were, on occasion, too focused on administrative issues as opposed to patient care
(FN 15), when utilized to discuss and address interprofessional practice concerns, this weekly meeting was seen as effective.

Time also posed a barrier to meaningful interpersonal interaction between professionals in the same clinic. While much brief, task-specific communication occurred, our observations revealed that the busy daily routine did not allow for any casual non-work related conversation, interprofessional or otherwise. A physician in site 2 explained,

[She] does not even see the other physicians on her team who are working at the same time...she has to [talk] with the nurse and secretary because they are working together... She wants to know the others but time is a major factor that impedes the social interaction.

(FN 09)

A resident from site 2 confirmed that

[Resident and nurse] interactions are generally brief and not very frequent...there is really little opportunity to interact with the clerks and the nurses because everyone is so busy.

(FN 14)

Across our research sites, non-medical health professionals generally interacted less frequently with doctors, nurses, and clerical staff than those groups did with one another, whether conversation was of a social or professional nature. Communication between doctors and dietitians, for example, began with a consultation request and in many cases did not involve any face-to-face exchange unless specifically requested by the patient. Allied health staff reported a desire to interact more with other health care providers in their clinics, but, as one physiotherapist remarked, 'The problem is that [I don't] really have many opportunities to get to know them' (site 2, FN 03).

By contrast, opportunistic social interaction was the norm in site 3, where staff described its significance for fostering a collaborative environment. This was especially true of the group in their former location, which was a badly maintained building in a disadvantaged area of the city. A physician from site 3 explained that the original clinic location was a very social place, and because we were so isolated from everything else, 
the staff would all get together at lunchtime and end up discussing the patients and administrative issues casually (FN 15). The informal way that business was conducted over lunchtime conversation was markedly absent from the group dynamic in their new clinical location, where the lack of a common lunchroom and changed staff schedules impeded such socialization. The same physician from above disappointedly remarked, 'The only time [we] eat together now is during [the weekly] team meetings' (FN 15). Similarly, a clerical staff commented that since the move, the clinic has lost some of the informality that worked well for communication with physicians. Since the clinic remains open during the lunch hour, which is a change from the old clinic's hours of operation, representatives from the clerical group and the two lab technicians have changed their schedules to accommodate for this. Now, she explained, issues are only raised when there is 'a problem' whereas before staff could talk about it informally at lunch (FN 15).

In all three sites, interprofessional collaboration was also impacted by the length of time the staff knew and worked with one another. Employment status played a role in perceived trustworthiness of others and opportunity for collaboration. In site 1, one-third of the nurses were neither permanent nor full-time and some staff perceived these nurses to be less invested and knowledgeable. A resident in site 1 stated that she only approached the two fulltime nurses who have been (in the clinic) a long time. She felt she could trust their judgments over the casual nurses because of their longevity and permanency (FN 14). This was not necessarily the view among more senior physicians (FN 13); however, there remained a perception that the clinic's staff was transitory. One senior physician in site 1 explained that the clinic had 'a business-like environment [where] you do your time and get out' (FN 18). This experience was in part due to the many 'part-timers' who worked here and 'all have other jobs' (FN 18).

The part-time presence of physicians in the clinics was a barrier to collaborating with other health care professionals. For instance, an occupational therapist in site 3 explained that she would like to work with one particular physician given their shared interest in palliative care, however, 'It may be difficult, since [the doctor] is only part-time' (FN 19). From a site 2 physician's perspective, working in the clinic only two days per week had led to her feeling 'pretty invisible' (FN 09). Additionally, she attributed this feeling to her low patient volume and the fact that she had not referred any patients to the (dietitian), physiotherapist, or other health care professionals (FN 09).

\section{Discussion}

\section{Interdependence of space and time}

Our fieldnotes suggest that time and space operate interdependently to impact interprofessional work, resulting in different local models of collaboration. We have identified such factors as team composition and size, duration of time worked and working together, regularity of team meetings, opportunistic interprofessional interactions, and proximity of providers to one another as key temporal and spatial components of interprofessional collaboration.

In site 1 , the negative impact of time, particularly in the form of part-time and casual employment of nurses, counteracted the potential advantages of nurses' co-location in the unit with all physicians. Co-location of team members has been argued to promote the 'boundedness' of teams and help to define them as 'a social entity' (Oandasan et al., 2006). Though nurses were physically close to physicians, thereby being available for frequent consultation or support, some physicians were reluctant to collaborate with those not having fulltime status. This was not the case in sites 2 and 3 where core team nurses were employed full-time. The effect of nurses' employment status on nurse-physician collaboration and teamwork in general has not been explored in-depth (Grinspun, 2007); however, a barrier to collaboration appears to exist where nurses do not hold full-time positions.

And co-location itself was not entirely desirable in site 1 as the current space was not entirely accommodating. Despite some physical separation of private offices and the nursing station, staff had very small individual workspaces as well as minimal shared space to communicate on either a formal or informal basis. Rather than promoting increased contact and collaboration, staff viewed this overcrowded, sometimes uncomfortable, spatial arrangement as resulting in poor understanding among interprofessional team members, invasions of privacy, and profession-specific silos. 
Monthly interprofessional meetings were introduced in site 1 to improve communication among the interdisciplinary group. Interprofessional team meetings have been shown to promote collaboration in several clinical settings (Molyneux, 2001), though not in the absence of additional supportive organizational factors such as appropriate team size, consistent team membership, and opportunity outside of meetings to interact (Xyrichis and Lowton, 2007; Baxter and Brumfitt, 2008). In site 1, team meetings had not yet proved successful in changing the formal business-type environment that participants described, neither had they lessened the psychic isolation experienced during everyday clinical work.

By comparison, in site 2, the small co-located core teams of physicians-nurse-secretary were collaborative and cohesive. In most cases, staff on core teams had worked together for a period of ten years or longer, and had shared their self-contained but relatively spacious clinical areas within the larger unit. Where the design of their private offices was not ideal, as was shown to be the case above with a nurse, staff members were able to share others' workspace, effectively enhancing communication with them. Interprofessional meetings were not built into daily schedules since the staff felt as though sufficient interprofessional communication took place spontaneously. Many preferred this method of communication because they did not have to wait to discuss any issues or concerns. Temporal and spatial factors thus combined here to create positive sub-team environments, although team-to-team interaction was infrequent.

Across the larger unit of site 2, however, it was the other health care professionals who experienced the negative impact of space. Length of time worked in the clinic was not a big enough factor to promote interprofessional collaboration where co-location was absent. With their private offices at a significant distance from the rest of the unit, these staff on the periphery did not have ample opportunity for collaboration and rarely engaged in opportunistic conversation with core team members. Even though some health care professionals had worked at the family health care centre for over a decade, their physical separation from the core teams could not be overcome and feelings of under-utilization and disconnect existed. Further, as a result of this physical and psychological distance, some of these staff believed that colleagues from other professions did not have a complete understanding of their scope of practice, their programmes, and services as evidenced by the lack of referrals they were sent. Some believed that attempts to educate others about their roles would be rejected, which deterred them from participation in interdisciplinary education sessions and rounds. This perception constituted a perpetual barrier to improving interprofessional teamwork. Indeed, research has shown that team members' perceptions of others' understanding and respect for their expertise enhances collaboration and can improve patient care (Dieleman et al., 2004).

Site 3 was unique, in that staff from across the professions had worked together here for quite a long time. Given their history and the overall structure of the site as a smaller health care centre, these individuals knew one another more intimately than those in our other two research sites. It was not uncommon for them to refer to their colleagues as 'friends' or 'family' including those from within different professions. Staff symbolically connected this sense of belonging to the group with their former clinical space, an unconventional informal unit that brought them together daily for lunch and casual conversation, work-related and otherwise. The regularity and informality of their weekly meeting helped staff foster existing relationships and forge relationships with newcomers. In their former location, collaboration across the professions was thus positively affected by the way time and space intersected.

Upon moving to a new, more 'clinical' health care unit, changes in schedules and structural reorganization of personnel and their offices had a negative outcome on both interprofessional relationships and the nature of interprofessional work. Staff lost their shared lunchroom and no longer spent their lunch in conversation with one another. Some individuals who were previously co-located were now divided by an elevator. From an organizational perspective, the laboratory and its two long-standing technicians were no longer considered part of the family health care unit. Technicians thus no longer attended team meetings. And lastly, whereas the former clinic would close over the lunch hour, the new clinic remained open. This meant that some members of the clerical staff were unable to attend the weekly interprofessional meeting, leaving them feeling excluded and not valued as team members. 


\section{Implications and conclusion}

The variability in team collaboration, which results from the interaction of temporal and spatial factors, has important implications for the transition of primary health care centres into FHTs. We found that providers in smaller interprofessional environments where providers are visible to one another and work from a reasonable proximity (not too far but not too close), are more interactive, both professionally and socially, and are more likely to collaborate effectively. This finding is important given the financial resources currently allocated to spatial reorganization and the expansion of existing small practices. Such plans for spending are best informed by systematic analyses of situated workplace relations such as the one provided here. Interprofessional team expansions, which may occur across wide geographical areas, might create frustrations and stress if not implemented with attention to this critical issue. If professionals are not brought together often enough and long enough to establish mutually appreciative working relationships and clear knowledge of one another's roles, they might fail to promote teamwork altogether.

The ethnographic method has allowed us to gain practical insight into communicative practices in the primary health care setting that are otherwise not accessible. Insights arrived at from this comparison of the impact of time and space suggest the need to strike a balance of quantity and quality of time devoted to interprofessionalism, and to design clinical space to maximize the quality and quantity of interprofessional connections.

Communication patterns and the nature of health professionals' relationships are influenced in important ways by the temporal and spatial arrangements of their work. For the successful transition of family health centres to interprofessional collaborative family health teams these factors must be seriously considered.

\section{Acknowledgements}

We would like to thank Health Canada, the Department of Family and Community Medicine and the University of Toronto for their support and participation in our study. Lorelei Lingard is supported by a CIHR New Investigator award and the BMO Financial Group Professorship in Health Professions Education Research.

\section{References}

Andrews, G.J. 2006: Geographies of health in nursing. Health \& Place 12, 110-18.

Andrews, G.J. and Evans, J. 2008: Understanding the reproduction of health care: towards geographies in health care work. Progress in Human Geography (in press online), 1-22.

Barrett, J., Curran, V., Glynn, L. and Godwin, M. 2007: Interprofessional collaboration and quality primary healthcare. Ottawa, Ontario: Canadian Health Services Research Foundation. Retrieved on 16 May 2008 from http://www. chsrf.ca

Baxter, S. and Brumfitt, S. 2008: Once a week is not enough: evaluating current measures of teamworking in stroke. Journal of Evaluation in Clinical Practice 14, 241-47.

Boudioni, M., McLaren, S.M., Woods, L.P. and Lemma, F. 2007: Lifelong learning, its facilitators and barriers in primary care settings: a qualitative study. Primary Health Care Research and Development 8, 157-69.

Department of Health. 2000: The NHS plan: a plan for investment, a plan for reform. London: Stationary Office.

Department of Health. 2004: The NHS improvement plan: putting people at the heart of public services. London: Stationary Office.

Dieleman, S.L., Farris, K.B., Feeny, D., Johnson, J.A., Tsuyuki, R.T. and Brilliant, S. 2004: Primary health care teams: team members' perceptions of the collaborative process. Journal of Interprofessional Care 18, 75-78.

Grinspun, D. 2007: Healthy workplaces: the case for shared clinical decision making and increased full-time employment. Healthcare Papers 7, (Suppl), 85-91.

Halford, S. and Leonard, P. 2003: Space and place in the construction and performance of gendered nursing identities. Journal of Advanced Nursing 42, 201-208.

Hammersley, M. and Atkinson, P. 1995: Ethnography: principles in practice, second edition. London: Routledge.

Hansson, A., Friberg, F., Segesten, K., Gedda, B. and Mattsson, B. 2008: Two sides of the coin: general practitioners' experience of working in multidisciplinary teams. Journal of Interprofessional Care 22, 5-16.

Health Canada. 2004: Interprofessional education for collaborative patient-centred practice. Retrieved on 16 May 2008 from http://www.hc-sc.gc.ca/hcs-sss/hhr-rhs/strateg/interprof/ index_e.html

Hummers-Pradier, E., Scheidt-Nave, C., Martin, H., Heinemann, S., Kochen, M.M. and Himmel, W. 2008: Simply no time? Barriers to GPs' participation in primary health care research. Family Practice 25, 105-12.

Liaschenko, J. 1994: The moral geography of home care. Advances in Nursing Science 17, 16-25. 
Molyneux, J. 2001: Interprofessional teamworking: what makes teams work well? Journal of Interprofessional Care $15,29-35$.

Meuser, J., Bean, T., Goldman, J. and Reeves, S. 2006: Family health teams: a new Canadian interprofessional initiative. Journal of Interprofessional Care 20, 436-38.

Oandasan, I., Baker, R., Barker, K., Bosco, C., D’Amour, D., Jones, L., Kimpton, S., Lemieux-Charles, L., Nasmith, L., San Martin Rodriguez, L., Tepper, J. and Way, D. 2006: Teamwork in healthcare: promoting effective teamwork in healthcare in Canada. Ottawa, Ontario: Canadian Health Services Research Foundation. Retrieved on 16 May 2008 from http://www.chsrf.ca

Ontario Ministry of Health and Long Term Care. 2002: Family health teams. Canada: Government of Ontario. Retrieved on 16 May 2008 from http://www.health.gov.on.ca/ transformation/fht/fht_mn.html

Ostbye, T., Yarnall, K.S.H., Krause, K.M., Pollak, K.I., Gradison, M. and Michener, J.L. 2005: Is there time for management of patients with chronic diseases in primary care? Annals of Family Medicine 3, 209-14.

Rapport, F., Doel, M.A. and Elwyn, G. 2007: Snapshots and snippets: general practitioners' reflections on professional space. Health \& Place 13, 532-44.

Rapport, F., Doel, M.A., Greaves, D. and Elwyn, G. 2006: From manila to monitor: biographies of general practitioner workspaces. Health (London) 10, 233-51.

Reeves, S. and Lewin, S. 2004: Hospital-based interprofessional collaboration: strategies and meanings. Journal of Health Services Research and Policy 9, 218-25.

Reeves, S., Russell, A., Zwarenstein, M., Kenaszchuk, C., Gotlib Conn, L., Doran, D., Sinclair, L., Lingard, L., Oandasan, I., Thorpe, K., Austin, Z., Beales, J., Hindmarsh, W., Whiteside, C., Hodges, B., Nasmith, L., Silver, I., Miller, K.-L., Vogwill, V. and Straus, S. 2007: Structuring communication relationships for interprofessional teamwork (SCRIPT): a
Canadian initiative aimed at improving patient-centred care. Journal of Interprofessional Care 21, 111-14.

Sanjek, R., editor, 1990: A vocabulary for fieldnotes. Fieldnotes: the makings of anthropology. Ithaca: Cornell University Press, pp. 92-121.

Shaw, A., de Lusignan, S. and Rowlands, G. 2005: Do primary care professionals work as a team: a qualitative study. Journal of Interprofessional Care 19, 396-405.

Soklaridis, S., Oandasan, I. and Kimpton, S. 2007: Family health teams: can health professionals learn to work together? Canadian Family Physician 53, 1198-199.

Tai-Seale, M., McGuire, T.G. and Zhang, W. 2007: Physician and patient behaviour: time allocation in primary care office visits. Health Services Research 42, 1871-894.

Thompson, C., Dalgleish, L., Bucknall, T., Estabrooks, C., Hutchinson, A.M., Fraser, K., Rien de Vos, R., Binnekade, J., Barrett, G. and Saunders, J. 2008: Nurses' risk assessment decisions: a signal detection analysis. Nursing Research 57, 302-11.

Thompson, D.S., O'Leary, K., Jensen, E., Scott-Findlay, S., O-Brien-Pallas, L. and Estabrooks, C.A. 2008: The relationship between busyness and research utilization: it is about time. Journal of Clinical Nursing 17, 539-48.

Wilson, D.R., Moores, D.G., Woodhead Lyons, S.C., Cave, A. and Donoff, M.G. 2005: Family physicians' interest and involvement in interdisciplinary collaborative practice in Alberta, Canada. Primary Health Care Research and Development 6, 224-31.

Xyrichis, A. and Lowton, K. 2007: What fosters or prevents interprofessional teamworking in primary and community care? A literature review. International Journal of Nursing Studies 45, 140-53.

Yarnall, K.S.H., Pollak, K.I., Ostbye, T., Krause, K.M. and Michener, J.L. 2003: Primary care: is there enough time for prevention? American Journal of Public Health 93, 635-41. 